\title{
Fluid-structure interaction in rocket engines
}

\section{Analysis of side-loads resulting from rigid body rotation}

\author{
Emmanuel Lefrançois \\ Université de Technologie de Compiègne \\ Laboratoire Roberval UMR CNRS 6253 \\ BP 20529 - 60205 Compiègne Cedex \\ emmanuel.lefrancois@utc.fr
}

\begin{abstract}
Numerical approaches to predict side-loads on over-expanded launcher engines, resulting from the aeroelasticity, are proposed in this study: a stability model and a fluidstructure model. The main idea is to offer a better understanding of the repercussions likely to appear from the aeroelastic coupling in terms of side loads resulting from the motion of the compression shock and that may be responsible of damage effects on the current engines. It is notably shown the existence of a given natural torsional frequency of the nozzle for which the measured side loads are maximum, phenomenon associated with a transversal wave in the flow between both walls. These studies aim to improve the only current aeroelastic stability model in over expanded nozzle.

RÉSUMÉ. Un modèle numérique de couplage fluide-structure est présenté, visant à améliorer la prédiction du niveau de charges latérales dans un moteur fusée sur-détendu (présence d'un choc stabilisé) et subissant des mouvements de corps rigides. Ces derniers peuvent en effet avoir un effet destructeur sur la tenue du moteur et sont aujourd'hui encore observés sur les moteurs actuels. Il y est montré l'existence d'une fréquence critique de rotation du moteur pour laquelle le niveau de charge observé est maximum et lié notamment à l'existence d'une onde transversale en aval du choc venant régulièrement percuter les deux faces internes du moteur. L'objectif à terme de ces travaux est d'améliorer le seul modèle de stabilité aéroélastique actuellement appliqué aux moteurs fusée.

KEYWORDS: fluid-structure interaction, side-loads, over expanded rocket nozzle, rigid body motions, finite elements.

MOTS-CLÉS : interaction fluide-structure, charges latérales, moteurs sur-détendus, mouvements de corps rigides, éléments finis.
\end{abstract}

DOI:10.3166/EJCM.19.637-652 @ 2010 Lavoisier, Paris

EJCM - 19/2010. Fluid-structure interaction, pages 637 to 652 


\section{Context and interests for the study}

\subsection{Industrial context}

The design process of a launcher engine requires to ensure both thrust capabilities and lightweight considerations, this latter being justified to increase payloads. Moreover, the control of the launcher trajectory requires to ensure a correct orientation of the engine by the means of actuators. Consequently a launcher engine may never be considered as a rigid organe but as a flexible one. Flexibility may then be due to local elastic deformation of the divergent part and to rigid body motions around the nozzle fixation point (called cardan axis) by considering the flexibility of actuators.

Undesirable effects are particularly strong at low-altitude flight where the external pressure is strongest and opposes itself to the flow. Amongst them, we may cite side load effects that are due to a loss of symmetry of the flow whose the origin is still kept out of understanding. Their effects may be strong enough to damage the engine and deviate the launcher trajectory.

This phenomenon is known for a long time since the first works according to this, date back to the twenties, notably with Prandtl, Meyer and Stodola who worked on over expanded jets (i.e. with presence of an internal separation shock) (Summerfield et al., 1954).

\subsection{Current investigations for the origins of side loads}

A launcher engine is designed to operate in adapted conditions (no internal compression shock). However we may cite several cases where the engine may be used in over expanded conditions :

- the progressive increase of the chamber pressure during the starting phase of a launcher engine,

- during validation phases they must be tested at sea level in vacuum test chamber where the main flow encounters an external residual pressure of a few milli-Pascal.

Three possible causes of appearance of the side load effects currently keep the attention of the researchers, the interest evocated by many laboratories of the European community being well expressed in reference (Act, 1998) :

- the pressure fluctuations in the separation and recirculation zones downstream the shock (Schwane et al., 2002; Wong, 2005),

- the transition between two kinds of separation flow, the free separation shock and the restricted separation shock This transition may occur during the start-up process. Both cases lead to different wall pressure distributions that may generate side loads if the symmetry is not ensured. This phenomenon has been experimentally and numerically observed (Frey et al., 1999; Östlung, 2002; Deck et al., 2004), 
- the aeroelastic coupling beween the flow and the flexible part of the nozzle (including rigid body movements due to control actuators) (Östlung et al., 2001; Lefrançois, 2005; Pekkari, 1993).

Reaching higher chamber pressure levels for higher performances requires to increase area ratios in nozzle design that may lead to mechanical structures more sensitive to interact with the flow and to generate side loads.

\subsection{Aeroelasticity for expanded nozzle}

Aeroelasticity has been studied for at least forty years from a theoretical point of view (Bisplinghoff et al., 1975; Dowell, 1975; Fung, 1958) and more recently a numerical approach has been developed and proposed (Farhat et al., 1995; Kondo et al., 1987; Piperno, 1994; Tallec et al., 1996). The development of coupled models for aeroelasticity is quite recent due to its multidisciplinary nature. Moreover, its application to rocket engines has rarely been studied (Lefrançois, 2005; Pekkari, 1993; Tuovila et al., 1968). Currently, three main approaches are possible to study aeroelastic effects of an engine due to over expanded conditions :

1) an experimental investigation (complex and expensive) (Östlung, 2002; Tuovila et al., 1968),

2) a numerical aeroelastic stability analysis with a model initialy developed by Pekkari's team (Pekkari, 1993) and improved to detect dynamical instabilities (Lefrançois, 2005; Lefrançois et al., 2000),

3) a complete fluid-structure interaction (FSI) model developed in a parallel environment. It generally consists of three codes, respectively dedicated to compute the structure deformation, the fluid flow and mesh deformation with messages passing in order to regularly update common data between all models (expensive in terms of CPU cost).

For a rigid nozzle with a flexibility only due to external mechanisms such as control actuators (rotation and translation), the Pekkari's stability model predicts frequency shifting with regards to the compression shock position along the nozzle. We illustrate on Figure 1 the results obtained from both models (stability and fluidstructure) for a case with only one degree of freedom (abbr. dof) in rotation and with two $d o f$, a rotation and a displacement respectively associated with different rigidities.

The $x$-axis corresponds to the initial compression shock position $\left(X_{s e p}\right)$ that is contolled by the pressure chamber $P_{c}$. The $y$-axis reports the corresponding aeroelastic frequencies predicted by the stability model (lines) and those extracted by FFT analysis (Fast Fourier Transform ) from fluid-structure coupled results (symbols).

For both cases, it is clear that no frequency shifting has been observed from fluidstructure calculations even if the stability model respectively predicted a static instability for the first case (frequency collapses to zero) and a dynamic one for the second case (frequencies coallescing). The main explanation lies in the fact that frequency 


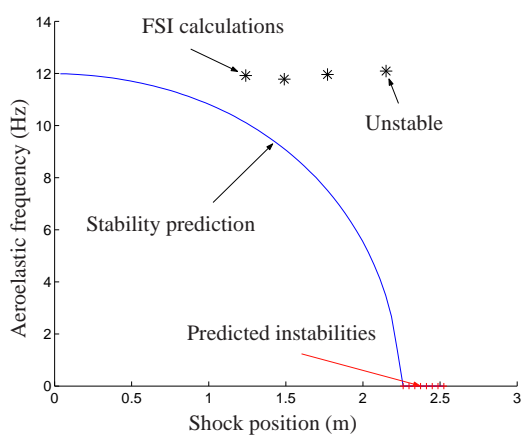

(a) 1 dof : rotation

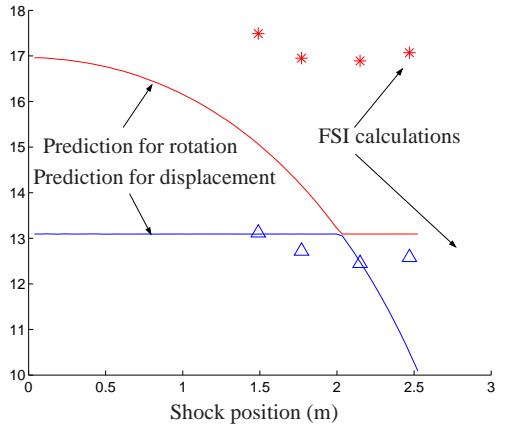

(b) 2 dof : rotation and $\mathrm{X}$-displacement

Figure 1. Frequency shifting predictions (lines) and FSI calculations (symbols)

shifting prediction may only be due to shock displacement (in regards with stability model). But for all cases, no shock motion has been reported during the fluid-structure calculations for this frequency range.

It is then necessary to better understand the interaction between a compression shock and a moving nozzle in order to be able to improve this stability model currently in used in the nozzle design.

The structure of this paper is decomposed in three parts. A first section describes the complete fluid-structure model. A second section exposes investigation ways to better understand the physics underlying between a shock in motion and nozzle movements. A section notably studies the influence of the natural frequency response of a nozzle in rotation on the shock excursion and points out the existence of a transversal wave for a precise frequency. Sensitivity analysis also permits to distinguish quasi-instataneous response of shock in response to the nozzle motion and dephased response. A last part concludes and presents ways currently investigated in order to improve the aeroelastic stability model.

\section{Numerical model for fluid-structure interaction}

This section briefly describes the fluid-structure interaction model used to study the effect of aeroelasticity on side loads for over expanded launcher engine (Lefrançois, 2005; Lefrançois et al., 2000). It consists of three codes, respectively dedicated to compute the structure deformation, the fluid flow and the mesh deformation. These codes are encapsulated in a parallel environment and interact within a coupling scheme. 


\subsection{Structure model}

A finite element technique is used for the space discretization of the flexible structure. The governing equations are based on the fundamental principle that may be written as :

$$
[M]\left\{\frac{\partial^{2} u}{\partial t^{2}}\right\}+\left\{f_{\text {int }}(u, t)\right\}-\left\{f_{\text {ext }}\right\}=0,
$$

obtained after assembling all over the mesh elements where :

- $[M]$ : global mass matrix,

$-\{u\}$ : nodal displacements vector,

$-\left\{f_{\text {int }}(u, t)\right\}$ : non-linear internal efforts,

$-\left\{f_{\text {ext }}\right\}$ : external forces resulting from aerodynamical coupling.

The main characteristics of the structure solver are :

- a Total Lagrangian Formulation is employed to calculate the deformations of a flexible structure under large displacements hypothesis ((Zienkiewicz et al., 2000), Vol. 2),

- the resolution of the resulting non-linear system is obtained using a NewtonRaphson iterative method (Dhatt et al., 2005; Zienkiewicz et al., 2000),

- an implicit Newmark-Wilson scheme is used for temporal resolution (Dhatt et al., 2005).

\subsection{Fluid flow model}

This model is based on the finite element resolution of the global set of equations governing inviscid and compressible fluid flow on a two-dimensional moving domain (Farhat, 1996; Lefrançois et al., 1999).

$$
\frac{\partial}{\partial t}\{J U\}+J\left(\frac{\partial\left\{F_{x}\right\}}{\partial x}+\frac{\partial\left\{F_{y}\right\}}{\partial y}\right)=0,
$$

where :

$-\{U\}$ : conservative variables,

$-\left\{F_{x}\right\}=\left\{F_{c x}\right\}-w_{x}\{U\}$ : convective flux along $x$-direction,

$-\left\{F_{y}\right\}=\left\{F_{c y}\right\}-w_{y}\{U\}$ : convective flux along $y$-direction,

with :

$$
\{U\}=\left(\begin{array}{c}
\rho \\
\rho u \\
\rho v \\
\rho e
\end{array}\right),\left\{F_{c x}\right\}=\left(\begin{array}{c}
\rho u \\
\rho u^{2}+p \\
\rho u v \\
(\rho e+p) u
\end{array}\right),\left\{F_{c y}\right\}=\left(\begin{array}{c}
\rho v \\
\rho u v \\
\rho v^{2}+p \\
(\rho e+p) v
\end{array}\right),
$$


with $\rho$ the mass density, $u$ and $v$ respectively the components of fluid velocity in $(x, y)$ system, $e$ the total energy per unit of mass and $p$ the local pressure given by the law of perfect gas :

$$
p=\rho \cdot r \cdot T=(\gamma-1)\left(\rho e-1 / 2 \rho\left(u^{2}+v^{2}\right)\right),
$$

with the temperature $T, \gamma=1.4$ and $r=287 u . S I$. The variables $\left(w_{x}, w_{y}\right)$ represent the local velocity components of the domain and $J(x, y, t)$ is the jacobian of the transformation between the movable physical space $(x, y)$ and a fixed reference space $(\xi, \eta)$. The main characteristics of the fluid solver are :

- an explicit Lax-Wendroff scheme is used for temporal discretization (Lax et al., 1960) with a shock capturing technique called Flux Corrected Transport (Boris et al., 1997) with a Zalesak's limiter (Zalesak, 1979) in zones where positivity is not ensured (presence of shocks for example). Stability is ensured using a CFL criterion (Cormack, 1992),

- a Discrete Geometric Conservation law is applied to ensure the same precision order and stability property of the solver obtained in the case of a rigid mesh (Farhat et al., 1995; Guillard et al., 2000),

- a decomposition domain technique is used to parallelize the flow solver in order to significantly reduce CPU cost. The partitioning tool CHACO (Hendrickson $e t$ al., 1995 ) is used to decompose the initial mesh on 'N' sub-meshes with nodes duplication on internal boundaries,

- the fluid mesh is deformed with a pseudo-material approach associated to a submesh technique (MSA for Moving Submesh Approach (Lefrançois, 2008)) to significantely reduce $\mathrm{CPU}$ cost.

\subsection{Coupling scheme between the fluid and structure codes}

The coupling must respect the following points :

- in order to preserve modularity aspects of each code taken separately, the coupling is done in establishing a 'message-passing' between solvers by using parallel tools offered by PVM (Parallel Virtual Machine) routines (Geist et al., 1994; Kessy, 1997),

- the exchanged data during calculations consist of the wall pressure distribution and time step from the fluid code to the structure one, and the update of the flexible boundaries common to both codes from the structure to the fluid,

- the characteristic times being different by several orders of magnitude between both codes (implicit structure code and explicit fluid code), the updating of wall conditions is made using a sub-cycling coupling scheme and thus carried out every $N_{f / s}$ fluid steps,

- the data exchange is here based on a discontinuous coupling scheme (see Figure 2 ), which although does not preserve the kinematic compatibility between the meshes 
of the fluid and the structure, makes possible to better ensure the transfer of energy and momentum between the two physics (transfer of a corrected pressure profile) (Piperno, 1994).

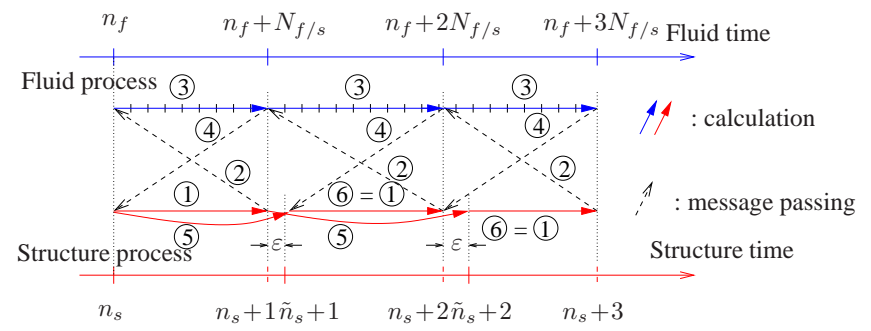

Figure 2. Discontinuous fluid-structure coupling scheme

The exchange process illustrated Figure 2 must be read as follows :

1) from time station $n_{s}$, we conduct a single time step for structure solver to update displacements, velocities, strains. New position corresponds now to time station $n_{s}+1$,

2) transfer to the fluid code of the informations based on the new structure state,

3) from time station $n_{f}$, we conduct $N_{f / s}$ consecutive fluid time steps to update all fluid data and reach time station $n_{f}+N_{f / s}=n_{s}+1$. The fluid mesh is progressively deformed in order to match both fluid boundary and new structure deformation,

4) transfer to the structure code of a corrected parietal pressure profile (Piperno, 1994),

5) structure deformation is then updated in accordance to these new fluid data in conducting a new calculation from previous time station $n_{s}$.

\section{Analysis of the shock response to forced rigid body rotation}

The objective here is to study the behaviour of the compression shock in response to a forced rotation regime imposed to an over expanded nozzle. It is motivated by the fact that the rotation around its cardan axis is one of the motion able to conduct to non-symmetric flows. Moreover, this motion is similar to the one encountered on real engine controled by actuators. A particular attention is then given to the evolution of the side loads calculated with :

$$
F_{x}=-\int_{n o z z l e} p(s) \vec{n}(s) \cdot \vec{i} d s,
$$

where $\vec{n}$ is the external normal vector and $\vec{i}$ the unity vector on the $x$-axis. The side loads results essentially from the compression shock motion $X_{\text {sep }}(t)$ that will be stored during all calculations. 


\subsection{Application case characteristics}

The main domain is illustrated on Figure 3b. It consists in a rigid 2D-nozzle with one degree of freedom (abbr. dof), defined by the rotation $\theta(t)$ around its cardan axis. The fluid domain (geometry and dimensions) with boundary conditions are explicitly illustrated Figure 3b.
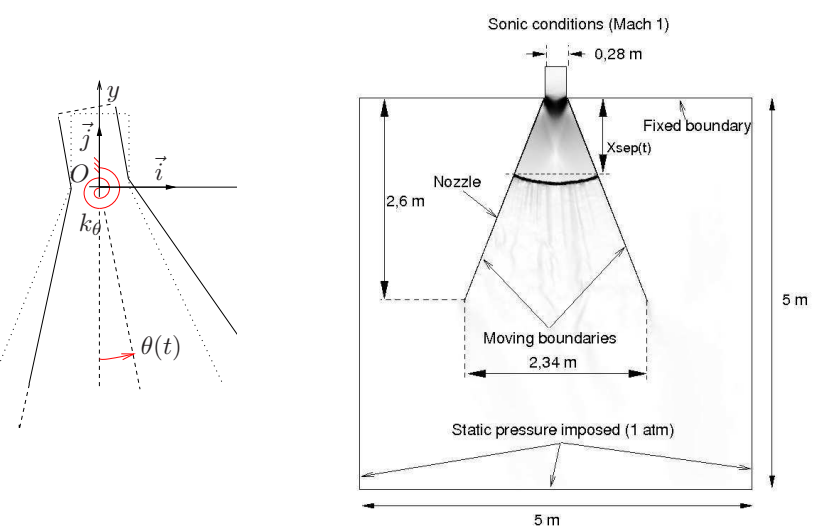

(a) Structure attached to cardan (b) Fluid domain and boundary conditions

Figure 3. Description of the fluid domain (dimensions, boundary conditions) and nozzle rigidities

In order to avoid local deformation of the nozzle, the Young's modulus has been choosen equal to $10^{18} \mathrm{~N} / \mathrm{m}^{2}$. The fluid flow is assumed inviscid and structural deformations are supposed linear (small perturbation theory). The fluid mesh is composed of 439073 triangular elements for 218083 nodes. The element size varies from 0.5 $\mathrm{cm}$ inside the nozzle with a progressive increase to $10 \mathrm{~cm}$ outside the nozzle. This progressive variation permits to numericaly diffuse the ignition shocks and consequently reduce the transient calculation time (see Figure 4) before starting the coupling phase. These diffusion effect is visible on the set of successive Schlieren (Kudryavtsev et al., 2001) on Figure 4.

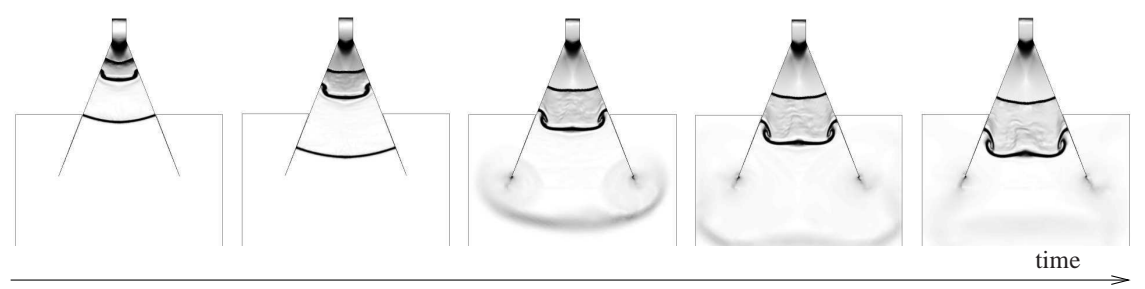

Figure 4. Ignition phase in a $2 D$ nozzle (inviscid flow) 
The structure mesh is extracted from the parietal boundaries of the fluid mesh (meshes conformity) and is composed of 1123 two-nodes beam finite elements (three dof per node, two displacement and one rotation).

Following the transition ignition of the nozzle, a forced regime in time of the rotation $\theta(t)$ of the nozzle is prescribed according to :

$$
\theta(t)=\theta_{o} \sin (\pi f t)^{2} \quad \text { with } \quad \theta_{o}=1^{o},
$$

where $f$ is the frequency of the forced regime. A square sinus form has been considered in order to progressively start the nozzle rotation such as $\dot{\theta}(0)=0$.

The nozzle pressure ratio (NPR : ratio $P_{c} / P_{\infty}$ ) equal to 3 leads to a compression shock stabilized at a distance of $X_{\text {sep }}^{o}=1 \mathrm{~m}$ after the throat.

\subsection{Results analysis}

A set of 16 FSI calculations has been performed for frequency values starting from $12 \mathrm{~Hz}$ to $150 \mathrm{~Hz}$. For each, 100000 fluid time steps have been conducted. The structure has been updated every 10 steps.

For each case the RMS (Root Mean Square) response of the shock motion $X_{\text {sep }}(t)$, given by :

$$
\operatorname{RMS}\left(X_{\text {sep }}\right)=\left(\frac{1}{t} \int_{0}^{t}\left[X_{\text {sep }}(t)\right]^{2} d t\right)^{1 / 2},
$$

is illustrated in Figure 5 for the considered frequency range.

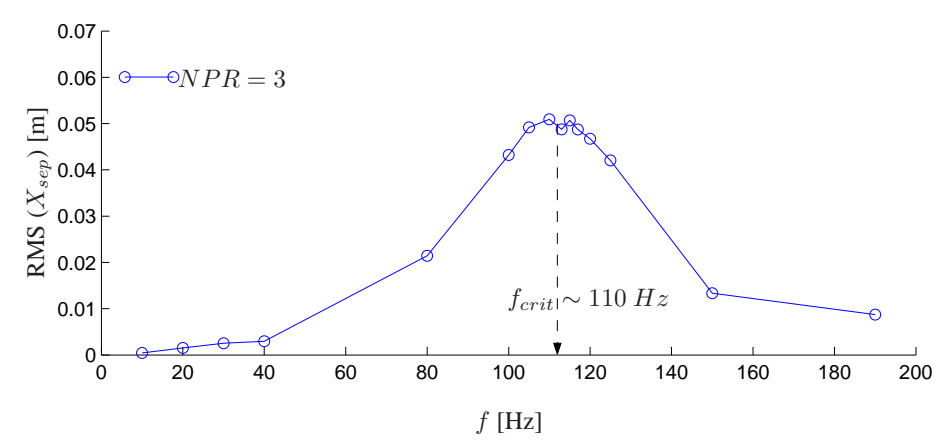

Figure 5. Sensitivity analysis of the shock motion with respect to $f$ (forced regime)

It can be observed that the shock motion depends on the frequency. This reveals three important distinct coupling regime : 
1) for low frequencies $(f<50 \mathrm{~Hz})$ the shock can almost be considered as fixed : the explanation may be found in the fact that the fluid characteristic time is lower than the structure period $(1 / f)$ permitting to the fluid to rapidly adapt itself with regards to moving environment. This also explains why the fluid-structure calculations did not confirm the frequency-shifting predictions, since Pekkari's model requires significant shock motions (see Section 1.3),

2) there exists a critical frequency ( $\sim 110 \mathrm{~Hz}$ for $N P R=3)$, corresponding to the maximum amplitude of the shock motion, that may lead to significant side-loads,

3 ) for high frequencies $(f>150 \mathrm{~Hz})$ shock and nozzle motions are not connected any more : for these cases, the fluid characteristic time is greater than the structure period $(1 / f)$ and fluid is not fast enough to adapt itself to moving environment.

In order to confirm the observations for the first and third regimes, new calculations have been conducted for a higher rotation value $\left(\theta_{0}=15^{\circ}\right)$. Results are respectively shown in Figure 6 for a low frequency and in Figure 7 for a high frequency. It confirms

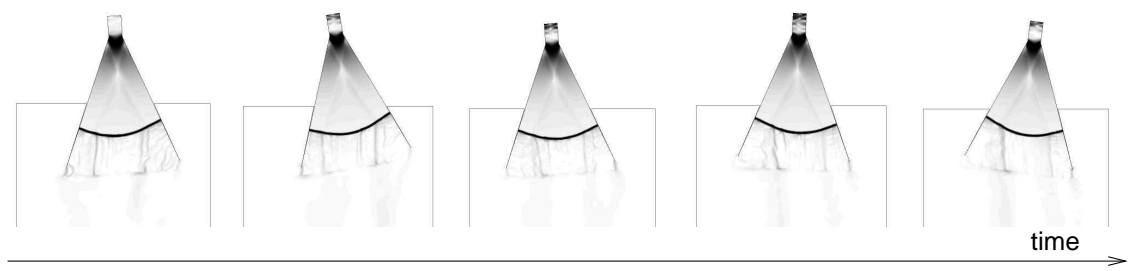

Figure 6. Sequences of numerical Schlieren : slow forcing rotation of a $2 D$ nozzle $(f<50 \mathrm{~Hz})$

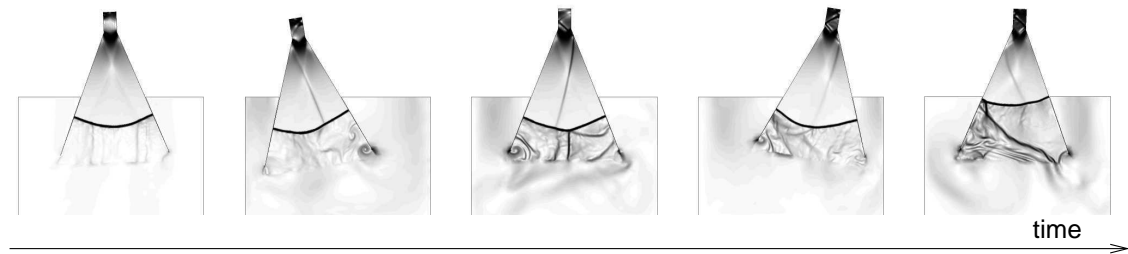

Figure 7. Sequences of numerical Schlieren : fast forcing rotations of a $2 D$ nozzle $(f>150 \mathrm{~Hz})$

for the lower frequency case that, even for a higher rotation angle, the fluid rapidly adapts itself if nozzle motion is relatively slow with regards to fluid characteristic time. For the second case, we observe that this adaptation capacity of the fluid flow is not valuable any more.

Figures $8 \mathrm{~b}$ and $9 \mathrm{~b}$ respectively illustrate the time signals of wall pressure for nodes respectively located upstream and downstream the shock. Their respective positions 
are given in Figures 8a and 9a. The $\mathrm{x}$-axis is the normalized time given by $t^{*}=t \times f$. The lower signal for each figure corresponds to the angular velocity $\dot{\theta}(t)$.

According to Figure $8 b$, the pressure evolutions are perfectly in phase with regards to nozzle angular velocity $\dot{\theta}$. Fluid rapidly adapts itself to the nozzle motion. This is obsserved regardless the frequency value.

For wall pressure evolutions extracted downstream the shock (see Figure 9b), we may observe a phase shift $\varphi$ between pressure and angular velocity that also depends on frequency. Parietal pressure seem to be governed by external flow conditions.
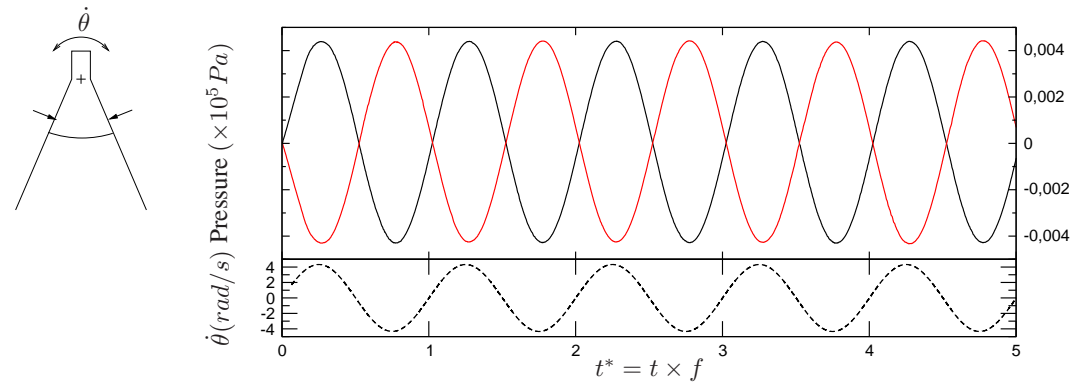

(a) Extractions

(b) Wall pressure signals

Figure 8. Wall pressure evolutions upstream the shock (right wall in gray, $80 \mathrm{~Hz}$ )
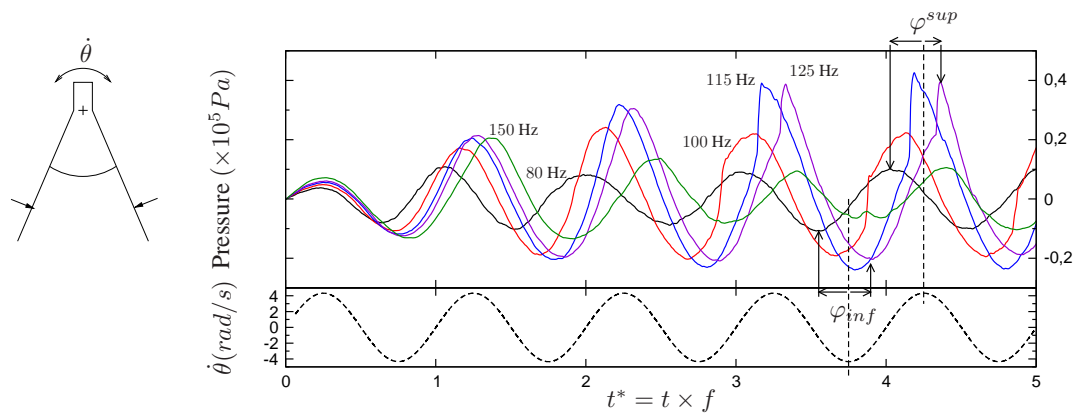

(a) Extractions

(b) Wall pressure signals

Figure 9. Wall pressure evolutions downstream the shock (right wall)

Transversal wave for $f=f_{\text {crit }}$ : piston analogy

For the critical frequency $f_{\text {crit }}=110 \mathrm{~Hz}$ corresponding to the maximum sideload value (see Figure 5), we observe the interesting phenomenon of a transversal wave that propagates itself between both opposite walls. This latter is self-induced by 
the nozzle motion as a resonance phenomenon. This effect is illustrated on the set of images given in Figures 10 and 11.

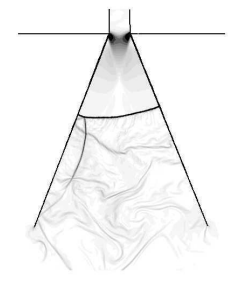

(a) $t^{*}=3.22$

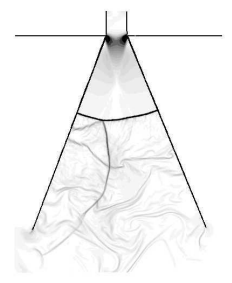

(b) $t^{*}=3.35$

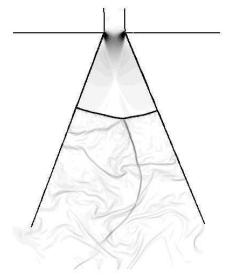

(c) $t^{*}=3.48$

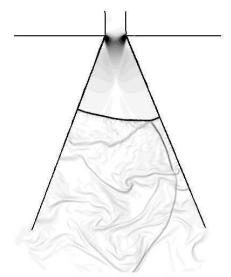

(d) $t^{*}=3.61$

Figure 10. Transversal wave propagating from the left to the right

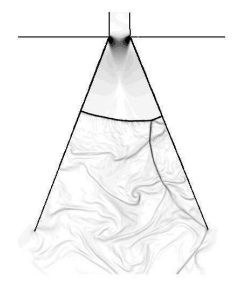

(a) $t^{*}=3.75$

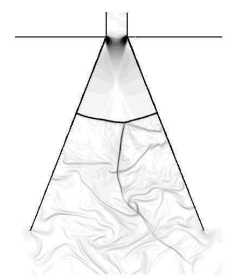

(b) $t^{*}=3.89$

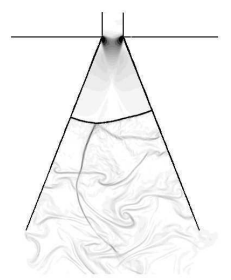

(c) $t^{*}=4.03$

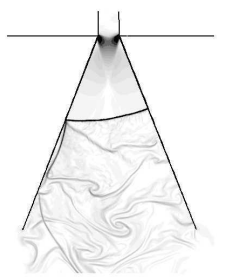

(d) $t^{*}=4.16$

Figure 11. Transversal wave propagating from the right to the left

The wave growth finds its origin in a single waves addition process, each wave resulting from each cycle of nozzle motion. This is analogous to the wave generation observed in a shock tube where both extremities would be associated to dependent movable pistons as illustrated in Figure 12a.

Characteristics theory (Délery, 2008) permits to confirm this process as the origin of the transversal wave that results from a resonance phenomenon between the nozzle frequency motion and the time required for a single wave to propagate between both extremities. Characteristic lines are plotted in Figures $12 \mathrm{~b}$ and $12 \mathrm{c}$ for a domain with left and right walls assimilated to pistons with a forced motion at 80 and $115 \mathrm{~Hz}$. The shock wave results from characteristics intersections and is clearly visible for the $115 \mathrm{~Hz}$ case, whereas the $80 \mathrm{~Hz}$ case does not let appear such a shock. 


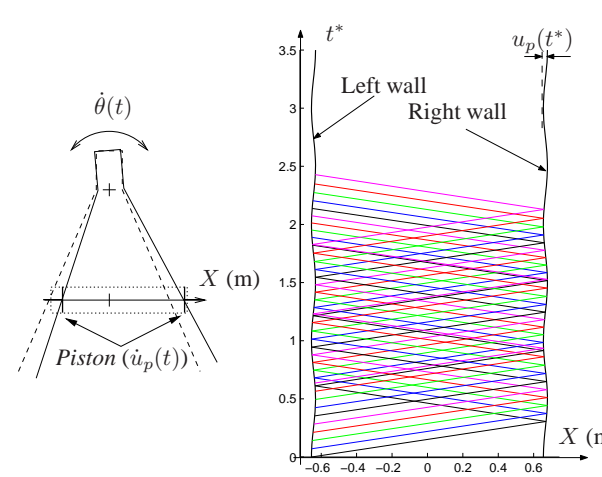

(a) Piston analogy

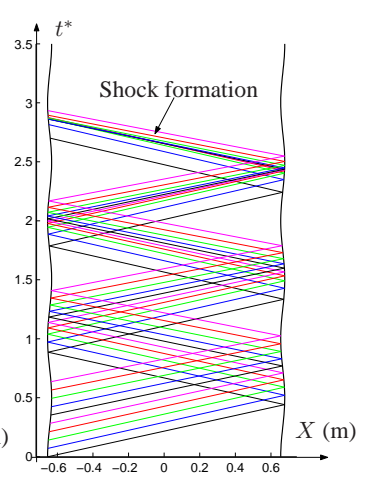

(c) $f=115 \mathrm{~Hz}$

Figure 12. Characteristics theory in a shock tube with moving extremities

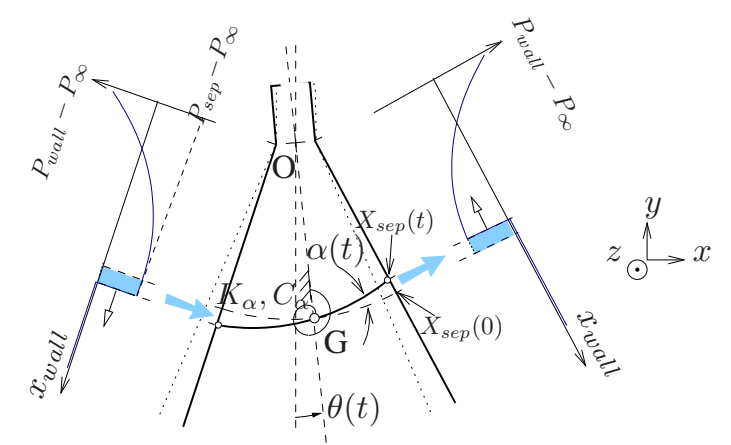

Figure 13. Mass-spring analogy for side-loads predictions resulting from shock motions

\section{Perspectives}

A numerical model for fluid-structure interactions has been presented for the study in a $2 \mathrm{D}$ over expanded nozzle, of the interaction between side loads and rigid body rotations. This model is based on three different solvers respectivey for fluid, structure and mesh deformation that are connected with a coupling scheme in a parallel environment.

It has been shown the existence of a critical frequency for the rotation of the nozzle for which the measured side loads are maximum and that also corresponds to the phenomemnon of a transversal wave that highly deforms the shock. It has also been observed that the compression shock may adopt a quasi-steady state response with regards to nozzle rotations for low frequencies, whereas it is not the case any more for 
higher frequencies for which a phase shift may be measured between side loads and angular velocity.

A direct application resulting from these studies is to improve the only current aeroelastic stability model in over expanded nozzle. This latter (Pekkari, 1993) predicts frequency shifting due to rigid body motions whereas a complete set of codes for fluid-structure interaction reveals none.

Studies and acquired knowledges exposed in this paper will permit to furnish a large amount of data that are currently in use to develop a model that is based on a mass-spring analogy. This analogy is essentially motivated by the shock behaviour with regards to the frequency of nozzle rotations and is illustrated in Figure 13 where the shock is assimilated to a pseudo mass with a single degree of freedom in rotation.

This analogy requires the determination of the pseudo rigidity from all calculations presented in this paper. This property extraction is currently under work for different value of NPR in order to study the effect of the initial shock position along the nozzle.

\section{Acknowledgements}

These works have been partly realized with the support of computer resources (PILCAM - Plateforme Inter-Laboratoires de CAlcul Distribués) financed as part of an ANVAR support and Heudyasic and Roberval laboratories (University of Technology of Compiègne, France).

The author is currently supported and financed by CNES (Centre National d'Études Spatiales) for studying aeroelasticity on flexible nozzle. This work is associated to a research group between CNES/ONERA/CNRS/SNECMA/ASTRIUM on jet flows in nozzle and after bodies of launchers.

\section{References}

Act, European Seminar on Rocket Nozzle Flows, octobre, 1998.

Bisplinghoff R. L., Ashley H., Principles of Aeroelasticity, Wiley and Son, New York, 1975.

Boris J. P., Book D. L., « Flux-Corrected Transport, I. SHASTA, a Fluid Transport Algorithm That Works », Journal of Comp. Physics, 135, pp.172-186, (original publication : 1973), 1997.

Cormack R. W. M., « Numerical Computation of Compressible Viscous Flow », February, 1992, Stanford University course.

Deck S., Anh T., «Unsteady side loads in a thrust-optimized contour nozzle at hysteresis regime », AIAA journal ISSN 0001-1452 CODEN AIAJAH, vol. 42, no 9, pp. 1878-1888, 2004.

Délery J., Traité d'aérodynamique compressible - Volume 3 : application de la théorie des caractéristiques et écoulements transsoniques, Lavoisier, 2008.

Dhatt G., Touzot G., Lefrançois E., Méthode des éléments finis, Hermès, 2005. 
Dowell E. H., Aeroelasticity of plates and shells, Noordhoff International Publishing, 1975.

Farhat C., « High Performance Simulation of Coupled Nonlinear Transient Aeroelastic Problems », Ecole d'Eté - Porquerolles (France) 1-6 Juillet, 1996.

Farhat C., Lesoinne M., Maman N., « Mixed explicit/implicit time integration of coupled aeroelastic problems : three-field formulation, geometric conservation and distributed solution», Int. J. for Num. Meth. in Fluids, Vol. 21, pp. 807-835, 1995.

Frey M., Hagemann G., « Flow Separation and Side-Loads in Rocket Nozzles », AIAA 99-2815, 1999.

Fung Y. C., An Introduction to the Theory of Aeroelasticity, Wiley and Son, New York, 1958.

Geist A., Beguelin A., al., PVM 3 User's Guide and Reference Manual, Technical report, Oak Ridge National Laboratory, May, 1994.

Guillard H., Farhat C., « On the significance of the geometric conservation law for flow computations frey on moving meshes », Comput. Methods Appl. Mesh. Engrg. 190, pp. 1467-1482, 2000.

Hendrickson B., Leland R., « The CHACO user's guide, version 2.0 », Sandia National Laboratories, 1995.

Kessy E., Decomposition de domaine et calcul parallele distribue : Application a la mecanique des fluides, PhD thesis, LMFN-CORIA, Université Rouen - France, 1997.

Kondo N., Tosaka N., al., « Numerical simulation for coupled system of viscous flow and elastic shell », Numerical Methods in Laminar and Turbulent Flow, Vol. 4, Part 2, p 1798, 1987.

Kudryavtsev A., Hadjadj A., « Visualisation graphique en mécanique des fluides », Flu Visu conference, Rouen (FR), June, 2001.

Lax P. D., Wendroff B., « Systems of Conservation Laws », Comm. Pure and Applied Mathematics, 13 : pp. 217-237, 1960.

Lefrançois E., « Numerical validation of a stability model for a flexible over-expanded rocket nozzle », International Journal for Numerical Methods in Fluids, Vol. 49, pages 349-369, 2005.

Lefrançois E., « A simple mesh deformation technique for fluid-structure interaction based on a submesh approach », Int. J. for Num. Meth. in Engineering, No 75, pages 1085-1101, 2008.

Lefrançois E., Dhatt G., Vandromme D., « Fluid-Structural Interactions with Applications to Rocket Engines », International Journal for Numerical Methods in Fluids, Vol. 30 - 1999, pages 865-895, 1999.

Lefrançois E., Dhatt G., Vandromme D., « Numerical Study of the Aeroelastic Stability of an Overexpanded Rocket Nozzle », Revue européenne des éléments finis, Vol. 9 - No 6/2000, pages 727-762, 2000.

Östlung J., Flow Processes in Rocket Engine Nozzles with Focus on Flow Separation and SideLoads, PhD thesis, Royal Institute of Technology, Dept. of Technology, S 100 - 44, Stockholm, Sweden, 2002.

Östlung J., Damgaard T., Frey M., « Side-load Phenomena in Highly Overexpanded Rocket Nozzles », 37th AIAA/ASME/SAE/ASEE, Joint Propulsion Conference and exhibit, July 811, Salt Lake City, 2001.

Pekkari L. O., « Aeroelastic Stability of Supersonic Nozzles with Separated Flow », AIAA, 29th Joint Propulsion Conference and Exhibit, Monterey, CA, 1993. 
Piperno S., Staggered time integration methods for a one-dimensional euler aeroelastic problem, Technical report, CERMICS, INRIA - Sophia-Antipolis, France, Décembre, 1994.

Schwane R., Wong H., Torngren L., « Validation of Unsteady Turbulent Flow Predictions for Over-expanded Rocket Nozzles », Proceedings of International Conference in CFD2, Sydney, Australia, Springer-Verlag, Berlin, July, 2002.

Summerfield M., Foster C., Swan W., « Flow separation in overexpanded supersonic exhaust nozzles », Jet Propulsion, vol. 24, n 9, p. 319-320, September, 1954.

Tallec P. L., Mouro J., Structures en grands déplacements couplées à des fluides en mouvement, Technical report, INRIA - No 2961, Août, 1996.

Tuovila W. J., Land N. S., Experimental Study of Aeroelastic Instability of Overexpanded Rocket Nozzle Extensions, Technical report, NASA TN D-4471, April, 1968.

Wong H., « Theoretical Prediction of Resonance in Nozzle Flows », Journal of Propulsion and Power, Vol.21, No.2, pp. 300-313., 2005.

Zalesak S. T., « Fully multidimensionnal flux-corrected transport algorithms for fluids », $J$. Comput. Phys., 31, p.335, 1979.

Zienkiewicz O. C., Taylor R. L., The finite element method, Vol. 1, 2 and 3, Fifth edition, Butterworth-Heinemann, 2000. 